\title{
MS03-P15 | Novel Biochemical Bases For Nuclear Factor I X (NFIX)
}

Lapi, Michela (Università degli studi di Milano, Milano (MI), ITA)

My PhD project focuses on Nuclear Factor I X (NFIX), which is a transcription factor belonging to the NFI DNAbinding proteins family. NFIX plays an essential role in the development of several organs, most importantly skeletal muscle. It has been shown that upon NFIX inhibition in a dystrophic mice model there is an amelioration in the pathological features of the muscular dystrophy. Thus, these data demonstrated that the absence of NFIX protects from the progression of the disease in vivo. This study shed a light on the possible use of NFIX as a genetic tool to slow down the progress of muscular dystrophy, which is still incurable.

On these bases, my project aims of purifying and solving the novel structure of NFIX alone and in complex with its palindromic DNA consensus sequence. The goal of the study is to find small molecules able to inhibit NFIX transcriptional function.

During my PhD, I managed to find an acceptable protocol for expression and purification of truncated constructs of NFIX via a prokaryotic expression system. I was also able to find crystallization conditions suitable for NFIX-DNA complex, but I still have to work on crystals optimization. Moreover, I performed several biophysical analysis to better characterize the binding of NFIX to the target DNA and the protein stability. 